\title{
Understanding the impact of failure modes of cables for the design of Turbo-Electric Distributed Propulsion electrical power systems
}

\author{
S. Nolan, C.E. Jones, P.J. Norman, S.J. Galloway \\ Department of Electronic and Electrical Engineering \\ University of Strathclyde \\ Glasgow, Scotland \\ steven.nolan@strath.ac.uk
}

\begin{abstract}
The turbo-electric distributed propulsion (TeDP) concept has been proposed to enable future aircraft to meet ambitious, environmental targets as demand for air travel increases. In order to maximize the benefits of TeDP, the use of high temperature superconductors (HTS) has been proposed. Despite being an enabling technology for many future concepts, the use of superconductors in electrical power systems is still in the early stages of development. Hence their impact on system performance, in particular system transients, such as electrical faults or load changes, is poorly understood. Such an understanding is critical for the development of an appropriate electrical protection system for TeDP. Therefore, in order to enable appropriate protection strategies to be developed for TeDP electrical networks an understanding of how electrical faults will propagate in superconducting materials is required. An understanding of how technologies that utilize these materials may experience failure modes in ways that are uncharacteristic of their conventional counterparts is also needed. This paper presents a dynamic electrical - thermal model of a superconducting cable, at an appropriate level of fidelity for electrical power system studies, which enables the investigation of failure modes of cables. This includes the impact of designing fault tolerant cables on the electrical power system as a whole to be considered.
\end{abstract}

Keywords-Turbo-Electric, Distributed-Propulsion, Future Aircraft, Superconductor, Electrical Fault

\section{INTRODUCTION}

For the past three decades air traffic has doubled every 15 years and the trend is expected to continue into the first half of the $21^{\text {st }}$ century [1]. In order to facilitate this increase in demand, it is anticipated that by 2034 there will need to be 32,600 new aircraft [1]. Alongside this, there are ambitious environmental and performance targets specified for future aircraft in terms of runway field length requirements, noise pollution, greenhouse gas emissions and fuel burn [2]. These aim to ensure that future air travel demands can be met whilst minimizing the negative impact on the environment.

TeDP is a concept which has been proposed as potential route to meet the ambitious performance targets set out in [2]. A key challenge of TeDP is that the propulsion of the aircraft is provided by electrically driven propulsor motors, rather than

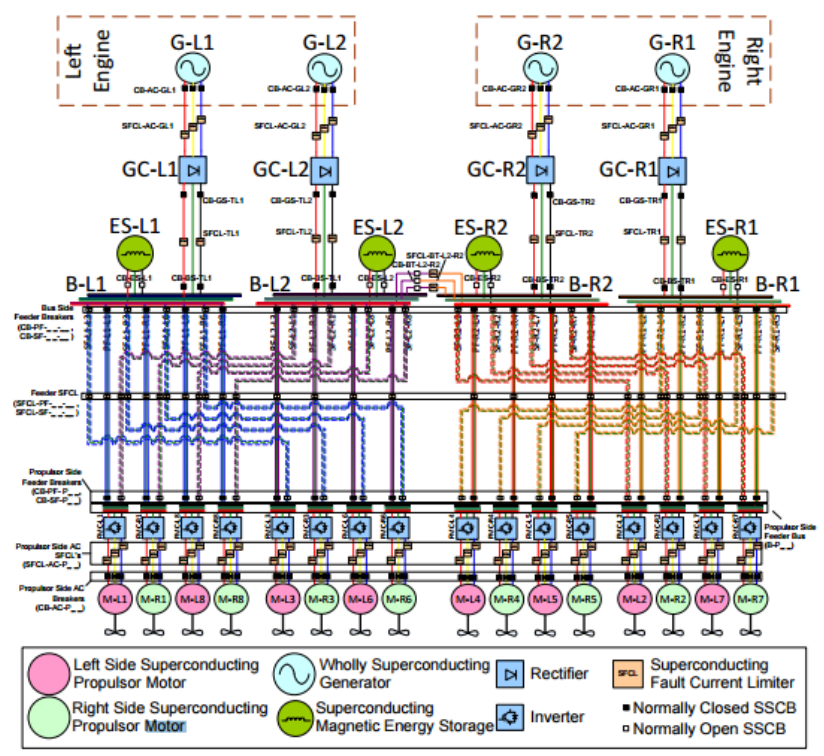

Figure 1. Example radial DC based TeDP architecture [3]

gas turbine engines. The electrical power is provided by generators driven by gas engines.

In order to have appropriately rated electrical machines with acceptable power densities for an aerospace application, it is proposed that superconducting electrical machines are used [4]. To minimize electrical losses and the weight of cabling it has been proposed that the electrical power system is, as far as technically possible, superconducting [3]. An example electrical network architecture that has been proposed for TeDP concept is shown in Fig.1 [3].

Central to the development of TeDP electrical power systems is the implementation of an appropriate protection and fault management system which will enable the aircraft to continue to fly safely in the event of an electrical fault occurring. In order to develop an appropriate protection system, the failure modes of network components, particularly in a physically compact, multi-terminal superconducting system (such as is proposed in Fig. 1) need to be assessed. Whilst it is acknowledged that the failure modes of all elements of a superconducting TeDP electrical power system require to be 
understood, the focus of this paper is on the failure modes of cables. Therefore, the paper first discusses some of the common issues that HTS cable designs need to address, the failure modes of conventional cables have also been considered. Secondly, in order to investigate cable failure modes in more detail the development of a thermal-electrical dynamic cable model is presented. This enables the specific case of the impact of DC cable failure on the electrical power system be studied and impact on power system design considered.

\section{CABLE FAILURE MODES}

\section{A. High-Temperature Superconducting cables}

Whilst superconducting technology has the potential to enable TeDP to be realized, the adoption of HTS materials into electrical components and the subsequent impact of these on the design and operation of the electrical power system is still poorly understood. In particular, the impact of HTS components on the dynamic stability of a network during both dynamic load changes and electrical fault conditions requires further study. Due to the negligible series resistance and much lower reactance of superconducting technologies, the inherent damping provided by the distribution network is much lower than systems using conventional conductors. This can have a significant impact on fault current levels which are expected to be as much as an order of magnitude higher than normal load current [5]. This is challenging because superconductors only remain in the superconducting state if their temperature, magnetic field strength, and current density are kept below a specific, critical threshold. If any of these values are exceeded, a phase change into the resistive state occurs. This is known as a quench condition.

When superconductors undergo a quench, the material exhibits a sharp rise in electrical resistivity. While this resistance can provide some damping to any unexpected current, the Joule heating that occurs during this process can lead to significant damage to HTS components [6]. Ideally the entirety of a superconductor would quench uniformly, allowing the full volume to absorb the fault energy. However inhomogeneity in the critical thresholds along the length of the material can lead to hotspots forming [7]. These locations then have to absorb a disproportionate amount of energy which can result in thermal take-off and localized damage over a short period of time [7]. Due to the relatively high heat capacity of HTS (compared to low temperature superconductors), the spread of the normal resistive area during a quench is in the order of $\mathrm{cm} / \mathrm{s}$, increasing the likelihood of damage from hotspots [8]. Improving the normal zone propagation velocity following a quench will be important in ensuring the stability of superconducting components.

Variations in the critical parameters of superconducting materials are caused by a number of factors related to both manufacturing practices and operating environment. The manufacturing of HTS involves inserting defects into the structure of the material to create flux pinning sites. These defects serve to prevent the movement of flux vortices within the material which would otherwise produce an electric field in the presence of a flowing current. This allows the critical current and magnetic field strength of HTS to reach very high levels. However, the process results in variations in the critical thresholds along the length of the conductor [7]. In the event of electrical faults occurring, these inconsistencies of the sensitivity of the material to current and magnetic field strength will result in certain sections of the material being at a greater risk of quenching and becoming hot spots. Hence a desirable identifiable attribute of protection systems for TeDP is that they are able to detect, and hence minimize, the impact of hotspots to ensure the longevity of superconducting equipment.

To prevent hotspots, many quench protection strategies spread the normal zone quickly to share the thermal burden equally throughout the volume of the material [9]. Excess current is dumped into shunt resistors. These goals are achieved through a combination of active and passive quench protection strategies [9]. For instance, superconducting magnets can achieve quench protection actively through the switching of radiators housed within the components structure that rapidly heat the material [9]. To protect superconducting cables a passive approach is generally adopted. This includes the use of either a low impedance parallel branch that can serve as an alternate path for current during fault conditions, referred to as fault tolerant cable design [10]. Conversely a high impedance material is used in a fault limiting design, which aims to reduce the fault current by increasing the overall impedance during quench [10]. These design approaches present a challenge for TeDP electrical power systems because a low impedance path requires a large cross-sectional area, increasing cable weight and volume, hence impacting on system fuel burn. Hence this diminishes one of the main advantages of HTS cables. Conversely, a high impedance path would require a very small cable area due to the relatively short distances of cable required on an aircraft (compared to a land based electrical power system), ultimately reducing the ability of the parallel path to withstand heat generation.

An alternative approach which may be considered is to utilize dedicated fault current limiters. However a disadvantage of fault current limiters compared to solutions which use the cable construction to regulate fault current is that fault current limiters must be placed at carefully chosen, specific points in the network. This exercise ultimately becomes a delicate trade of network coverage and system weight

\section{B. Stability}

From the discussions presented in Section II A, it is clear that in order to measure the capability of the network to maintain satisfactory operation following a disturbance, it is necessary to evaluate both thermal and electrical stability criteria. In nonsuperconducting conductors, resistive damping provided can have a significant effect on system transients, both during nonfault and fault scenarios. For instance, such resistive damping has a significant impact on the settling time of these switching transients [11]. An example of the impact of electromagnetic transients on a superconducting multi-terminal systems is investigated by the authors of [12]. The authors demonstrate that a change in load conditions at a single terminal results in transient oscillations between adjacent power-electronics modules. These oscillations may remain on the system for significant periods of time due to the lack of electrical resistance 
and may ultimately be detrimental to power quality [12]. In order to reduce the impact of switching transients within superconducting networks there are a number of methods presented in the literature that can be used for both AC and DC systems. For instance, the use of a damping circuit consisting of a resistor in parallel with an inductor can be shown to provide damping for transient oscillations in superconducting HVDC systems [11].

The stability of a superconducting system can also be measured by considering the ability of components to maintain superconducting properties under different operating conditions. In low temperature superconductors (LTS) the ability of the material to maintain the superconducting state is typically measured through current and temperature margin. These coupled margins determine the maximum tolerable increase in temperature or current before quench occurs. While these design margins can give indications of the stability for a superconductor following a disturbance, they may not be suitable for HTS due to the loss mechanisms that affect steady state temperature in HTS [13]. This is an important distinction as short-term overcurrent conditions that heat the superconductor up can reduce the amount of time the components can support a given current until the cooling system reduces the temperature to the pre-disturbance steady state [14]. This could also have the effect that protection requirements will need to be adjusted according to the systems' thermal as well as electrical conditions.

\section{CABLE MODEL}

In order to ensure that system transients will not lead to excessive electrical and thermal stress, a dynamic cable model is required which considers both the electrical and thermal elements. In addition, such a model is required to have sufficient level of detail to inform at an electrical protection and system design level, without requiring excessive processing resources.

A number of complex methods for modelling superconducting cables and materials have been presented in the literature. For example, the Finite Element Method (FEM) and Finite Difference Method (FDM) can be used to calculate the complex heat sharing between different layers of the material [15]. These are not well suited to dynamic system studies due to their computational intensity. Therefore in order to model the transient behavior within a system, this paper models the cable as a non-linear resistance that varies according to the E-J power law (1); an approach demonstrated for developing dynamic models of resistive fault current limiters $[15,16]$.

At current levels below the critical current level for a cable, flux pinning prevents the vast majority of flux vortices (quanta of flux that penetrate the bulk of the HTS material) from moving in response to the flowing current and thereby producing an electric field according to Faraday's Law. However there will still be a very slight field strength due to flux-creep. This produces an electric field strength according to (1), where $\alpha$ is the flux-creep coefficient, $J(t)\left(\mathrm{A} / \mathrm{m}^{2}\right)$ is the current density with $J_{c}(T)\left(\mathrm{A} / \mathrm{m}^{2}\right)$ being the temperature dependent critical current, $E_{c}(\mathrm{~V} / \mathrm{m})$ is the critical electric field strength [17].

$$
E(J, T)=E c\left(\frac{J(t)}{J_{c}(T)}\right)^{\alpha}
$$

Once $J_{c}(T)$ is exceeded, the flux vortices escape their pinning sites as the Lorentz force exerted on them by the moving current exceeds the pinning strength of the material. This state is called flux-flow and the resultant movement of flux vortices creates an electric field strength [7]. It is shown in [16] and [17] that under these conditions the electric field strength can be modelled for Bi-2223 according to (2), where $J_{c r}\left(\mathrm{~A} / \mathrm{m}^{2}\right)$ is the critical current density at a given reference temperature, $E_{o}(\mathrm{~V} / \mathrm{m})$ is the electric field strength at the transition between flux-creep and flux flow and $\beta$ is the flux-flow coefficient [16].

$$
E(J, T)=E_{o}\left(\frac{E_{c}}{E_{o}}\right)^{\frac{\beta}{\alpha}}\left(\frac{J_{c r}}{J_{c}(T)}\right)\left(\frac{J(t)}{J_{c r}}\right)^{\beta}
$$

Following this transition, there is a sharp increase in electric field strength and the Ohmic heating within the superconductor increases significantly. This continues until the temperature of the superconductor rises above the critical threshold or the depairing current is exceeded. The de-pairing current is taken as the point at which the effective resistance due to flux-flow exceeds the material's normal resistivity [18]. This causes an immediate quench as it becomes more favourable for electrons to travel conventionally through the material [20]. Following the transition into the resistive state, the resistance of the conductor becomes only dependent on temperature, as indicated in (3) where $T(\mathrm{~K})$ is the operating temperature and $\rho(\Omega / \mathrm{m})$ is the electrical resistivity at the critical temperature $T_{\mathrm{c}}(\mathrm{K})$.

$$
E(J, T)=\rho\left(\frac{T}{T_{c}}\right) J(t)
$$

In addition, the critical current density, $J_{c}$, is dependent on operating temperature of the superconductor: where $T_{r}$ is the temperature at the reference critical current density [15-16].

$$
J_{c}(T)=J_{c r}\left(\frac{T_{c}-T(t)}{T_{c}-T_{r}}\right)
$$

Losses within high temperature superconductors originate from two primary sources: eddy currents induced in the metallic sheath and the superconducting hysteresis effect. At frequencies below $1 \mathrm{kHz}$, hysteresis is the dominant form of power loss [19]. Hence at this stage eddy currents have been neglected. It is acknowledged that future versions of the model may need to consider eddy currents due to the expected inclusion of power electronic converters, with switching frequencies of $5 \mathrm{kHz}$ [3].

The superconducting hysteresis effect occurs due to the flux pinning within superconductors. When a magnetic field is applied to a HTS, it penetrates the material and becomes polarized in the direction of the applied field. When the applied field is then reversed, work must be done to overcome the flux pinning and polarize the field in the opposite direction [19]. This effect was included in the model by applying Norris's equation for hysteresis losses in elliptical geometry. This has been shown to be a good approximation for losses in Bi-2223 multifilamentary cables [19]. This is given in (5) where $P(\mathrm{~W} / \mathrm{m})$ is power loss, $i$ is the ratio of operating current relative to the critical current of the material, $f(\mathrm{~Hz})$ is the operating frequency 
and $\mu_{o}\left(\mathrm{mkgs}^{-2} \mathrm{~A}^{-2}\right)$ is the permeability of free space and $I_{c}$ is the critical current.

$$
P=\frac{f I_{c}^{2} \mu_{o}}{\pi}\left((1-i) \ln (1-i)+(2-i) \frac{i}{2}\right)
$$

The cable is constructed of multiple layers, each of which presents an inherent specific heat capacity, thermal conductivity and volume which will influence the movement of heat flux through the cable. Hence, the calculation of instantaneous temperature of the cable is challenging. It was considered sufficient at this stage to model the thermal aspect of the cable as an adiabatic model of heat transfer between the cable and the coolant. Hence, the temperature is calculated using (6) where $T_{\mathrm{a}}$ $(\mathrm{K})$ is the ambient temperature, $C_{s}\left(\mathrm{~J} / \mathrm{m}^{3} \mathrm{~K}\right)$ is the volumetric specific heat capacity. $Q_{\text {in }}(\mathrm{J})$ and $Q_{\text {out }}(\mathrm{J})$ describe the heat generated in the superconductor and the heat transferred to the cryogen.

$$
T(t)=T_{a}+\frac{1}{C_{s}} \oint\left(Q_{\text {in }}(t)-Q_{\text {out }}(t)\right) d t
$$

The heat dissipated in the superconductor is calculated using the Joule heating relationship considering current sharing between the superconducting layer and shunt stabilizer. The heat flux removed from the superconductor into the coolant is calculated using Fourier's law for heat conduction applied to a cylindrical geometry (7).

$$
Q=-2 k \pi r l \frac{d T}{d r}
$$

The simplification of the thermal model leads to a number of limitations within the model. The temperature distribution within the variety of material layers and the impact of the flow of the coolant on the variation in temperature across the length of the cable have been overlooked at this stage. Both may have an impact on thermal stress, and hence failure, of the cable. However due to the relatively long time constant of the heat transfer process relative to the expected time constant of the DC electrical system [20], the simplified adiabatic model presented in Equation 7 is adequate as an approximation for the purposes of investigating the impact of a transient event on the electrical power network [21].

\section{CASE Study}

\section{A. Overview}

By inspection of the DC architecture presented in Fig. 1, it can be seen that this network is a multi-terminal DC network, with converters interfacing $\mathrm{AC}$ loads and sources onto the network. The model developed in Section III was used to investigate the electrical and thermal response of the cable to a rail to rail $\mathrm{DC}$ fault.

Following the application of the rail to rail short, the transient response of the superconductor is expected to follow one of the following:

- The superconductor exceeds the critical current and is able to assist in dissipating the fault energy before the critical temperature is reached. This would allow the cable to resume the superconducting state when the circuit is re-energized, although the maximum operating current may be limited until the cooling system removes the thermal energy created during the fault.

- The superconductor exceeds the critical current and subsequent heating forces the temperature to rise above $T_{c}$, this provides large damping to the fault current however the cable will require a period of cooling before normal operation can resume.

- If the superconductor does not quench then the electrical energy will circulate through the fault path and the rectifier diodes until the small flux-creep and resistance of the diodes dissipate the energy.

The circuit model is implemented as shown in Fig. 2. The inductance of the cable was set to $1 \mathrm{mH}$ and the critical current of the superconductor is taken as $1000 \mathrm{~A}$. The voltage across the capacitor is also kept constant at $4.5 \mathrm{kV}$ [3]. The sensitivity of the response of the two different system parameters (filter capacitance and stabilizing resistances) was investigated.

\section{B. Results}

Figs. 3 and 4 show the fault current response and superconductor temperature respectively for a range of scenarios in which the stabilizing resistance, $\mathrm{R}_{\text {stable, is held constant at } 100}$ $\mathrm{m} \Omega$ and the DC link capacitance $\left(\mathrm{C}_{\mathrm{f}}\right)$ is varied between $50 \mathrm{mF}$, $225 \mathrm{mF}$ and $500 \mathrm{mF}$. These results show that as the filter capacitance increases, the amount of energy dissipated by the capacitor and the temperature of the cable in response to the fault also both increase.

Fig. 5 shows the peak temperature reached in the superconductor for variations in the filter capacitance (from 0 to $250 \mathrm{mF}$ ) and shunt resistor (from $11 \mathrm{~m} \Omega$ to $100 \mathrm{~m} \Omega$ ). This figure indicates that the size of the shunt resistance is a key factor in determining whether the cable can recover the superconducting state for any given capacitance discharge event.

Fig. 6 displays the peak current experienced for a range of values of filter capacitance (from 0 to $250 \mathrm{mF}$ ) and shunt resistor (from $0 \mathrm{~m} \Omega$ to $100 \mathrm{~m} \Omega$ ). It can be seen that increasing the stabilizing parallel resistance can have a positive effect on reducing the size of the fault current. Conversely increasing the capacitance increases the peak fault current experienced by the cable. 


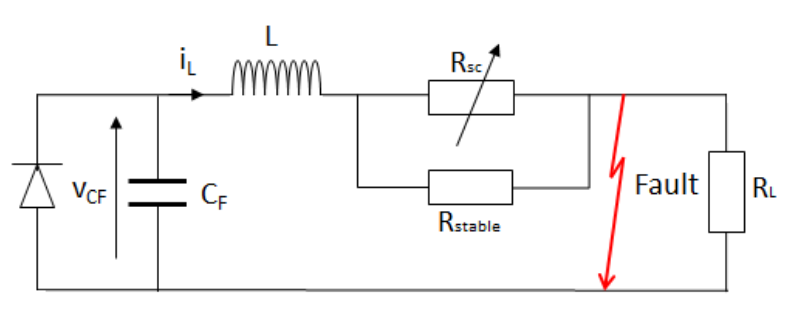

Figure 2. Circuit Diagram of Fault Case

\section{Discussion}

The case study presented demonstrates the potentially serious impact of a discharging DC link capacitance on superconducting cable behaviour. The risk of causing a quench condition is highlighted. This may cause damage to the cable as the energy stored in the capacitor is dissipated as heat within the superconductor. Even if no permanent damage is caused to the cable, the quench may remove the cable from service for a significant period of time (seconds to minutes) [22], before the cable recovers and is cooled to below the critical temperature, Tc. Depending on the network redundancy and fault management strategy, this removal may significantly impact on system performance.

Whether the superconductor undergoes a quench is dependent on either the discharge current of the capacitor exceeding the de-pairing current or being sustained for long enough at a sufficient level that the temperature of the cable exceeds the critical temperature level. Whether the superconducting cable is able to provide useful damping without exceeding $T_{c}$ is dependent on the length of the cable, as this increases both the resistance of the superconductor during the flux-flow and normal states as well as providing a larger volume to dissipate the resulting heat. Increasing the area of the superconducting cable provides a larger volume to dissipate the fault energy but reduces damping provided by the cable as the critical current density, $J_{c}$, will also increase. Alternatively, if the cable is designed to handle the maximum fault current without quenching this could lead to the solid state switching components within the converter being forced to dissipate the energy discharged by the capacitor.

Figure 3. Current Response for Different Values of DC link capacitance

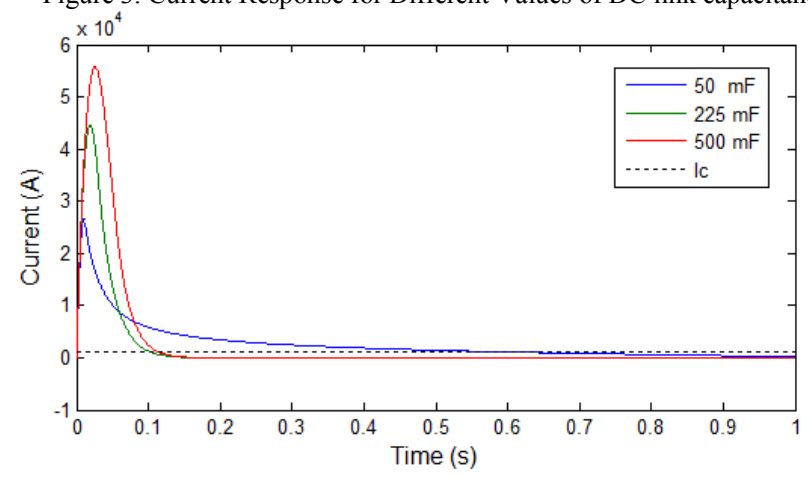

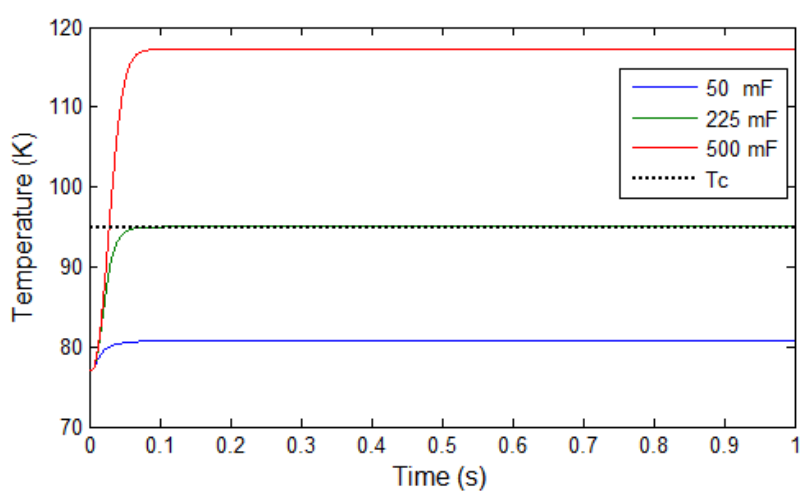

Figure 4. Temperature of superconductor following DC link capacitor discharge.

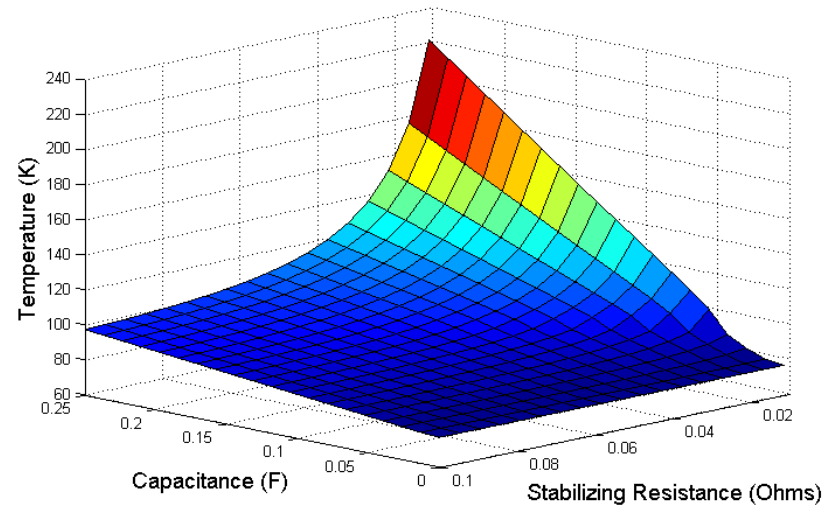

Figure 5. Temperature Following Capacitor Discharge with Varying Stabilizing Resistance and Capacitance Values

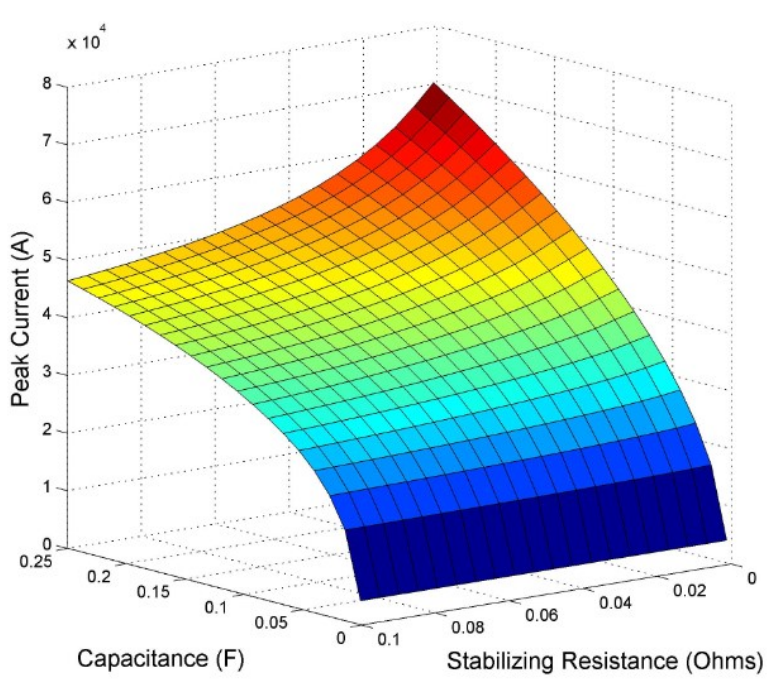

Figure 6. Impact of Capacitance and Stabilizing Resistance on Peak Fault Current

Appropriate overrating of these components may significantly increase the weight and efficiency penalty attributable to the power electronic converters, which is highly undesirable as it is known that without overrating, the cooling requirements of the 
power electronics present a significant challenge [23]. An alternative may be to consider different converter topologies with current blocking capabilities. Alongside this, it is clear that there is strong coupling between the failure modes, design and choice of components and the choice of protection system.

\section{CONCLUSION}

This paper has explored failure modes of a superconducting DC cable through the use of a thermal-electrical cable model. The results demonstrated how the cable may be designed to be less likely to fail, but that these design choices result in a series of trades, which link system stability and component failure with protection, redundancy and architecture design choices. It is clear that in order to develop appropriate TeDP power systems, it is not adequate to only understand the failure modes of the cable. The failure modes of other components and devices within the system also require characterisation. This will enable informed trades concerning design and choice of components, architecture, protection and redundancy for TeDP electrical power systems to be made.

\section{ACKNOWLEDGMENT}

This work was undertaken as part of the Rolls- Royce UTC program.

\section{REFERENCES}

[1] Airbus, "Flying By Numbers", Airbus, [online] Available: http://www.airbus.com/company/market/forecast/, 2015, accessed on: $12^{\text {th }}$ of May 2016.

[2] J. L. Felder, H. D. Kim, and G. V. Brown, "Turboelectric Distributed Propulsion Engine Cycle Analysis for Hybrid-Wing-Body Aircraft", 47th AIAA Aerosp. Sci. Meet. Incl. New Horizons Forum Aerosp. Expo., 2009.

[3] M. J. Armstrong et al., "Architecture, voltage, and components for a turboelectric distributed propulsion electric grid", NASA/CR - 2015218440, [online] Available: http://ntrs.nasa.gov/archive/nasa/casi.ntrs.nasa. gov/20150014237.pdf, 2015, accessed on: $12^{\text {th }}$ May 2016.

[4] H. Kim, "Distributed Propulsion Vehicles", 27th Int. Congr. Aeronaut. Sci., pp. 1-11, 2010.

[5] C. E. Jones, K. Davies, P. Norman, S. Galloway, G. Burt, M. Armstrong, and A. Bollman, "Protection System Considerations for DC Distributed Electrical Propulsion Systems Protection", SAE AeroTech Congr. \& Exhibition, pp. 1-10, 2015.

[6] I. Chew, A. Lapthorn, P. Bodger and W. Enright, "Superconducting transformer failure: Testing and investigation", Australian Universities Power Engineering Conference, , pp. 1-5, 2009.

[7] W. T. B. de Sousa, "Transient Simulations of Superconducting Fault Current Limiters", Ph.D. dissertation, Dept. Elect. Eng, Federal University of Rio de Janeiro, Brazil, 2015.

[8] D. Colangelo and B. Dutoit, "Impact of the normal zone propagation velocity of high-temperature superconducting coated conductors on resistive fault current limiters", IEEE Trans. Appl. Supercond., vol. 25, no. 2, pp. 1-7, 2015.

[9] P. F. Smith, "Protection of superconducting coils", Rev. Sci. Instrum., vol. 34, no. 4, pp. 368-373, 1963.

[10] C. E. Bruzek, A. Allais, K. Allweins, D. Dickson, N. Lallouet, and E. Marzahn, "Using superconducting DC cables to improve the efficiency of electricity transmission and distribution (T\&D) networks", Superconductors in the Power Grid, Elsevier Ltd, 2015.

[11] N. Kimura, T. Funaki, and K. Matsu-ura, "Damping of Current Oscillation in Superconductive Line Applied for High Voltage Direct Current Transmission System", IEEE Trans. Appl. Supercond., vol. 3, no. 1, pp. 4-6, 1993.
[12] D. I. Doukas, Z. D. Blatsi, A. N. Milioudis, D. P. Labridis, L. Harnefors, and G. Velotto, "Damping of electromagnetic transients in a superconducting VSC transmission system", IEEE PowerTech Conference, 2015.

[13] A. Ishiyama and H. Asai, "A stability criterion for cryocooler-cooled HTS coils", in IEEE Transactions on Applied Superconductivity, vol. 11, no. 1, pp. 1832-1835, 2001.

[14] K. Sivasubramaniam, T. Zhang, A. Caiafa, X. Huang, M. Xu, L. Li, E. T. Laskaris, and J. W. Bray, "Transient capability of superconducting devices on electric power systems", IEEE Trans. Appl. Supercond., vol. 18 , no. 3, pp. 1692-1697, 2008.

[15] W. T. B. De Sousa and M. Noe, "The ADI method for simulations of SFCL”, IEEE Trans. Appl. Supercond., vol. 25, no. 2, 2015.

[16] S. M. Blair, C. D. Booth, and G. M. Burt, "Current - Time Characteristics of Resistive Superconducting Fault Current Limiters", IEEE Trans. Appl. Supercond., vol. 22, no. 2, pp. 1-5, 2012.

[17] P. Manohar and W. Ahmed, "Superconducting fault current limiter to mitigate the effect of DC line fault in VSC-HVDC system", 2012 Int. Conf. Power, Signals, Control. Comput. EPSCICON 2012, 2012.

[18] C. M. Rey and A. P. Malozemoff, "Fundamentals of superconductivity", Superconductors in the Power Grid, Elsevier Ltd, 2015.

[19] S. Stavrev, "Modelling of High Temperature Supraconductors for AC Power Applications", Comp. and Comm. Faculty, École Polytechnique Federale De Lausanne, 2002.

[20] K. M. Davies, P. J. Norman, C.E. Jones, S.J. Galloway and M. Husband, "Examining the fault behaviour of a superconducting DC network", Dev. Power Syst. Prot. (DPSP 2014), 12th IET Int. Conf., 2014.

[21] S. M. Blair, "The Analysis and Application of Resistive Superconducting Fault Current Limiters in Present and Future Power Systems", Ph.D. dissertation, Dept. Elect. Eng, Strathclyde University, UK, April, 2013.

[22] M. Noe and M. Steurer, "High-temperature superconductor fault current limiters: concepts, applications, and development status", Supercond. Sci. and Tech., vol. 20, no.3, 2007

[23] C. E. Jones, P. J. Norman, S. J. Galloway, M. J. Armstrong and A. M. Bollman, "Comparison of Candidate Architectures for Future Distributed Propulsion Aircraft", IEEE Trans. Appl. Supercond vol. 26, no.6, 2016. 